Relations industrielles

Industrial Relations

\title{
W.B. GOULD : Japan's Reshaping of American Labor Law. Cambridge, Mass., The MIT Press, 1984, 193 pp., ISBN 0-262-07091-X
}

\section{Pierre Verge}

\section{Volume 39, numéro 3, 1984}

URI : https://id.erudit.org/iderudit/050072ar

DOI : https://doi.org/10.7202/050072ar

Aller au sommaire du numéro

Éditeur(s)

Département des relations industrielles de l'Université Laval

ISSN

0034-379X (imprimé)

1703-8138 (numérique)

Découvrir la revue

Citer ce compte rendu

Verge, P. (1984). Compte rendu de [W.B. GOULD : Japan's Reshaping of American Labor Law. Cambridge, Mass., The MIT Press, 1984, 193 pp., ISBN

0-262-07091-X]. Relations industrielles / Industrial Relations, 39(3), 642-643.

https://doi.org/10.7202/050072ar

Tous droits réservés (C Département des relations industrielles de l'Université Laval, 1984
Ce document est protégé par la loi sur le droit d'auteur. L'utilisation des services d'Érudit (y compris la reproduction) est assujettie à sa politique d'utilisation que vous pouvez consulter en ligne.

https://apropos.erudit.org/fr/usagers/politique-dutilisation/ 
According to Thompson, the policy taken by the unions becomes more and more obsolete. Trade union actions inhibit class consciousness and reflect work divisions. The experience of working people today is much different than long ago, when Marx formulated his analysis of the transformation of the class in-itself to the class in-itself. «The working class has to be subject to cultural, ideological and political factors outside the immediate sphere of production which act on the ability of highly divided workers to find common interests and a unity of purposey (p. 236). In the labour movement emphasis should be put on prefiguring new relations of production by subverting and changing forms of hierarchy and organization of production (p. 244). New forms of bargaining and industrial democracy should be encouraged as a more viable politics of production which integrates resistance of workers with transformation of the workplace reality. The passivity of traditional trade unionism in this new field is a sign of weakness and immaturity when taking into consideration the rapidly changing world of work.

\section{Alexander J. MATEJKO}

University of Alberta

\section{Japan's Reshaping of American Labor Law,} by W.B. Gould, Cambridge, Mass., The MIT Press, 1984, 193 pp., ISBN 0-262-07091-X

L'auteur, professeur à Standford Law School, avait présenté il y a deux ans une excellente vue d'ensemble du contenu et de la mise en oeuvre de la législation américaine du travail: A Primer on American Labor Law. Des séjours prolongés au Japon l'incitent cetie fois à comparer les principaux aspects du droit et des institutions du travail de ce pays aux situations correspondantes dans le sien. Le contenu américain de l'ouvrage est en effet important, l'objectif demeurant néanmoins la présentation de l'autre système.
L'occupation américaine du Japon sous l'autorité du général Mac Arthur devait entraîner l'adoption, en 1945 et 1946, de grandes lois du travail présentant certaines analogies avec le droit américain. Mais, l'ensemble du système japonais actuel se démarque profondément de ce dernier: telle est, pourraiton dire, la thèse de l'auteur. Le titre qu'il donne à son ouvrage masque en partie cette réalité, selon ses propres propos: «... the Department of State's decision led to Japanese labor legislation - major portions of which resemble American labor law, although the resemblance is one of form rather than substance and although the similarities must be seen in light of Japan's historical and continued adherence to the civil-law tradition, particularly that of Germany and France.) (p. 19)

Les premières pages décrivent d'une façon générale la perception japonaise du rôle du droit et du judiciaire dans la société, le phénomène syndical, axé le plus souvent sur l'entreprise, la diffusion de l'autorité dans celle-ci, l'importance de l'emploi permanent dans la grande entreprise, la propension au consensus, qui favorise la consultation, mais non l'arbitrage ... On fait ensuite un bref historique de l'adoption des trois grandes lois du travail de l'après-guerre, sans allusion véritable à la situation antérieure: la loi sur les syndicats, régissant les rapports collectifs du travail, celle mettant en place les commissions de relations de travail, chargées non seulement de voir à la répression des atteintes au droit d'association, mais également de régir une importante activité de conciliation et, enfin, celle portant sur les normes minimales du travail, qui, au-delà de ses prescriptions explicites, oblige les employeurs à déterminer de façon détaillée les conditions de travail.

Si certains aspects de cette législation rappellent le droit américain, par exemple, l'énoncé de pratiques déloyales de travail à la charge des employeurs, en particulier le refus d'engager la négociation collective, la similitude n'est que partielle. D'abord, 
d'autres éléments diffèrent profondément: ainsi en est-il de l'absence de monopole de représentation des salariés en faveur du syndicat majoritaire, pivot de la législation américaine, ou encore, de l'absence de formulation de pratiques déloyales, cette fois à l'encontre des syndicats.

Mais, c'est avant tout dans son fonctionnement quotidien que le système japonais révèle son identité: le rôle des commissions de relations du travail, la nature des ordonnances qu'elles rendent, deux sujets décrits en détail. Les comportements relatifs à la sécurité d'emploi sont également particuliers et semblent faciliter l'adaptation aux changements technologiques et économiques: aménagement de la mise à la retraite précoce, passage d'employés à des filiales, transmission de l'information de la direction de l'entreprise aux syndicats. On notera en particulier la nécessité, imposée par le judiciaire, de motiver le licenciement, qu'il s'agisse d'une situation disciplinaire individualisée ou d'un licenciement collectif économique. Devant de telles questions, l'auteur n'hésite pas à se montrer critique des lacunes qu'il constate en droit américain: "The Japanese have moved unabashedly toward the provisions of job security and of the information on which managerial decisions are based. American labor law on the other hand, has tended to genuflect to the concept of management prerogatives. Incursions into the realm of managerial autonomy are being made through both law and collective bargaining, but as of yet they are tentative and uncertain.» (p. 116)

Le système japonais est assurément moins conflictuel que le système américain. L'affrontement n'en est cependant pas toujours absent; il peut même à l'occasion se faire violent ou plus subtile, comme en témoigne l'activité judiciaire relative au «Ribbon Struggle», c'est-à-dire aux diverses insignes des salariés qui dénoncent l'employeur avec lequels ils sont en conflit. La grève n'est pas toujours efficace, le syndicalisme à l'échelle de l'entreprise permettant souvent à l'employeur de trouver des substituts en dehors de celle-ci. Les systèmes américain et japonais, enfin, ne sont pas dissemblables en ce qui a trait à l'admissibilité du lock-out défensif et à l'interdiction de la grève dans le secteur public.

Cette mise en parallèle des droits américain et japonais du travail, qui n'est ici que très incomplètement évoquée, se révèle, en définitive, un moyen utile de faire ressortir les traits marquants du second système: entreprise encore innovatrice dans les pays occidentaux; entreprise réussie dans le présent cas, semble-t-il, grâce à une remarquable aptitude à la synthèse de l'auteur.

Pierre VERGE

Université Laval

La sécurité d'emploi dans l'industrie de la construction au Québec, un rêve impossible?, Claudine Leclerc et Jean Sexton, P.U.L., 1983, XVI, 275 pp.,

ISBN 2-7637-7028-2

L'industrie de la construction est fort importante dans l'économie québécoise. L'analyse économique s'appuie même (peutêtre davantage qu'elle n'ose probablement l'avouer!) sur un adage: «ainsi le bâtiment va, ainsi va l'économie...».

Cette activité économique est si particulière qu'au Québec (et sous quelques autres juridictions, quoiqu'à un degré moindre) un régime de relations du travail particulier trouve application à ce secteur industriel.

On doit donc souligner avec d'autant plus d'éclat et de fanfare toute publication scientifique qui permet de mieux comprendre une partie fort importante de l'industrie de la construction, soit son régime de relations du travail et celui de la gestion de ses ressources humaines.

L'ouvrage récent de Claudine Leclerc et de Jean Sexton représente à cet égard une contribution importante à l'étude de cette industrie, quant à un aspect névralgique et 